\title{
Evaluación de la Nueva Definición de Politrauma en una Cohorte de Pacientes de 10 Hospitales Argentinos
}

\author{
${ }^{1}$ Ezequiel Monteverde, ${ }^{2}$ Laura Bosque, ${ }^{3}$ Betina Lartigue, ${ }^{4}$ Emilio Maciá, ${ }^{5}$ Cristian Barbaro, ${ }^{6}$ Claudio Ortiz \\ ${ }^{7}$ Enrique Ginzburg, ${ }^{8}$ Jorge Neira
}

\section{RESUMEN}

Introducción: La definición de Berlin de politrauma (2014) combina la severidad lesional con al menos uno de cinco complementos (edad, TAS, GCS, KPTT o EB).

Hasta ahora hay insuficiente información sobre cómo afecta esta nueva definición la identificación de los grupos con mayor mortalidad.

El objetivo de este trabajo fue evaluar la performance de esta definición comparándola con otras mediciones.

Materiales y métodos: Análisis retrospectivo observacional de ingresos prospectivos al Registro de Trauma de Fundación Trauma en 10 hospitales entre 2010-2016. Los criterios de inclusión fueron edad > 15 años y tener información completa para el cálculo de los índices. Los pacientes se compararon según las definiciones de politrauma (PT), trauma múltiple (Tmult), trauma mayor por ISS (TM_ISS) y trauma mayor por NISS (TM_NISS). Se analizaron medidas de performance.

Resultados: Se analizaron 259 hechos con PT, 802 con Tmult, 1349 con TM_ISS y 2143 con TM_NISS. El grupo PT resultó heterogéneo, con una mortalidad entre $44 \%$ y $71 \%$ según diferentes combinaciones de complementos. El 75\% tuvo lesiones por transporte y el $76 \%$ tuvo al menos una lesión encefálica AIS $>2$. El desenlace estuvo relacionado con el estado al ingreso, por impacto fisiológico (RTS) y por severidad lesional (ISS-NISS). La relación O/E para sobrevida por TRISS fue 0.73 .

La comparación con las otras definiciones mostró diferencias estadísticamente significativas en mortalidad pero no en ISS, NISS ni RTS. La evaluación de performance mostró que TM_NISS tuvo la mayor sensibilidad y el mayor VPN, mientras

\footnotetext{
1,2,6 Directora, ${ }^{3-5}$ Médico Monitor, ${ }^{7}$ Professor, ${ }^{8}$ Presidente

${ }^{1}$ Departamento de Registro de Trauma, Fundacion Trauma Buenos Aires, BA, Argentina

${ }^{2}$ Departamento de Dirección, Fundación Trauma, Buenos Aires BA, Argentina

${ }^{3-5}$ Departamento de Registro, Fundación Trauma, Buenos Aires Buenos Aires (Ciudad), Argentina

${ }^{6}$ Departamento de Dirección de Hospitales, Ministerio de Salud La Plata, Buenos Aires, Argentina

${ }^{7}$ Department of Surgery, Ryder Trauma Center, Miami, Florida United States

${ }^{8}$ Departamento de Consejo, Buenos Aires, Buenos Aires (Ciudad), Argentina
}

Corresponding Author: Ezequiel Monteverde, Directora Departamento de Registro de Trauma, Fundacion Trauma Buenos Aires, BA, Argentina, Teléfono: +1152791520 , e-mail: emonteverde@fundaciontrauma.org.ar que PT tuvo el mayor VPP. Como medida global, TM_ISS tuvo una precisión superior al resto (89\%) y una especificidad de $97 \%$.

Conclusiones: La definición de PT para identificación de pacientes con mayor riesgo de muerte no mostró ser superior a otras definiciones de uso corriente. Consideramos que la definición de PT necesita ser validada en estudios multicéntricos antes de recomendarse como un nuevo standard.

Palabras claves: Politrauma, Registro de trauma, Trauma, Trauma mayor, Trauma múltiple.

How to cite this article: Monteverde E, Bosque L, Lartigue B, Maciá E, Barbaro C, Ortiz C, Ginzburg E, Neira J. Evaluación de la Nueva Definición de Politrauma en una Cohorte de Pacientes de 10 Hospitales Argentinos. Panam J Trauma Crit Care Emerg Surg 2017;6(3):182-189.

Source of support: Nil

Conflict of interest: None

\section{ABSTRACT}

Introduction: Berlin definition of polytrauma from 2014 combines injury severity with at least one of five ancillary parameters (age, systolic blood pressure, Glasgow coma score, coagulopathy and acidosis). Until now there is insufficient evidence of this definition's capability to identify higher risk of mortality patients. The objective of this investigation was to evaluate this definition's performance to identify severely injured patients as compared with other current measures.

Materials and methods: Retrospective observational analysis was done on prospectively admitted patients to Fundación Trauma Registry in 10 Argentine hospitals between 2010 and 2016. The inclusion criteria were age $>15$ years and complete data for scores calculation. Patients were compared across four definitions: polytrauma, multiple trauma (MulT), major trauma by major trauma_injury severity score (MT_ISS), and by major trauma_new injury severity score (MT_NISS). Performance measures were applied.

Results: We identified 2143 cases meeting MT_NISS definition, 1349 for MT_ISS, 802 for MulT and 259 for polytrauma. Polytrauma group was heterogenous, with a mortality rate ranging from $44 \%$ to $71 \%$ (resulting from different component combinations). About $75 \%$ were transport-injured and $76 \%$ had at least one AIS3+ head injury. Hospital outcome was related to condition at admission, physiologic impact revised trauma score (RTS) and injury severity (ISS-NISS). Observed to predicted survival ratio method trauma and injury severity score (TRISS) was 0.73 . The comparison with the other definitions showed statistically significant differences in mortality but not in ISS, NISS, and RTS. Performance evaluation showed that MT_NISS had the highest sensibility and negative predictive value and

Presented in the General Competetion, PTS Congress, Maceio, Brazil in November 2016 
polytrauma had the highest predictive value (PPV). MT_ISS had the highest precision (89\%) and a specificity of $97 \%$.

Conclusion: Polytrauma definition used to identify patients with the highest probability of death did not show any benefit when compared with other current measures. We consider that this definition needs to be validated in multicentric studies before being recommended as a new standard.

Keywords: Definition, Multiple trauma, Score, Severity, Trauma.

\section{INTRODUCCIÓN}

Son múltiples las definiciones dirigidas a caracterizar al paciente con trauma severo. Esta situación ha sido descrita como "dos o más lesiones significativas," "al menos dos lesiones severas en la cabeza, el tórax o el abdomen o una de ellas asociada a una lesión en alguna extremidad" 2 y "dos o más lesiones, una de las cuales es potencialmente fatal." Adicionalmente, se la menciona como trauma múltiple, ${ }^{4}$ lesión mayor ${ }^{5}$ o trauma severo. ${ }^{6}$ Estos enfoques múltiples hacen que cuando se hace mención a alguno de ellos no se pueda asegurar cuál es el criterio utilizado. ${ }^{7}$

En el año 2014 Pape et al publicaron ${ }^{8}$ una definición de consenso revisando el término politrauma y basándose en el Registro de Trauma Alemán. Esta definición, más allá de incluir dos lesiones graves, incluyó también una serie de criterios accesorios para ayudar en la caracterización de los otros factores, más allá de las lesiones específicas, que condicionan el desenlace de los pacientes.

Más allá de los cambios en las definiciones que tienen que ver con incluir en las mismas algunos componentes de impacto fisiológico ${ }^{8,9}$ es muy probable que estas definiciones recientes estén incluyendo también algunos cambios como resultado de la mejora de la calidad de atención en países desarrollados. ${ }^{10,11}$

Esta nueva definición, si bien siguió un proceso sólido en su revisión, no fue validada en otras regiones y no fue respaldada por comités de trauma de sociedades científicas, lo que llevó a una difusión insuficiente.

Con el objetivo de evaluar el impacto de esta definición en una muestra de pacientes con características diferentes, se evaluó el Registro de Trauma de la Fundación Trauma de Argentina, que está activo desde 2010, contando actualmente con 10 hospitales, para evaluar el impacto de esta nueva definición, en comparación con otras definiciones de trauma severo usadas previamente.

\section{MATERIAL Y MÉTODOS}

Análisis retrospectivo observacional de ingresos prospectivos al Registro de Trauma de Fundación Trauma en 10 hospitales de la provincia de Buenos Aires, Argentina, entre 2010-2016. La provincia de Buenos Aires es una de las 23 provincias de la Argentina. Tiene un territorio de $307571 \mathrm{~km}^{2}$ y una población de 16.6 millones de habitantes. Esta provincia tiene una estrecha relación con la ciudad de Buenos Aires (capital del país), con la cual conforma una región denominada área metropolitana. El Programa de la Fundación Trauma se desarrolla en esta área metropolitana desde principios de 2010 y actualmente comprende 11 hospitales y 2 sistemas prehospitalarios de emergencias. Al momento del cierre de la muestra para este análisis, 10 hospitales estaban activos registrando pacientes en el Registro de Trauma (RT).

Se llevó a cabo un estudio observacional usando datos deidentificados de pacientes ingresados de forma prospectiva y sucesiva al RT de la Fundación Trauma en 10 hospitales de la provincia de Buenos Aires, Argentina entre 2010 y 2016. El RT incorpora pacientes que cumplen con la definición de trauma de la Organización Mundial de la Salud $(\mathrm{OMS})^{12}$ y permanezcan más de 23 horas en los hospitales o que fallezcan, sean derivados o egresen sin alta médica antes de ese tiempo. El RT codifica a través de AIS, CIE, GOS, RTS, ISS, NISS y TRISS.

Los criterios de inclusión fueron tener una edad mayor a 15 años y contar con información completa para el cálculo de los índices. Los pacientes se compararon según las definiciones de politrauma (PT), trauma múltiple (Tmult), trauma mayor por ISS (TM_ISS) y trauma mayor por NISS (TM_NISS).

Las variables continuas se trataron según su distribución y se expresan como media y desvío standard (DS) o mediana y rango intercuartil (RIC). La distribución de las variables categóricas se expresa como frecuencias y porcentajes. Las diferencias entre medias se estudiaron según test T de Student y, en caso de medianas, se utilizó el test de rangos de Wilcoxon (siempre que se cumpliera con los supuestos de distribuciones similares en ambos grupos y tendientes a la simetría). La asociación entre variables categóricas se estudió mediante el test de $\mathrm{Chi}^{2}$ con o sin corrección de continuidad de Yates, según correspondiera, o test de Fisher si la frecuencia esperada en alguna celda fuera menor a 5 . Se usaron medidas de performance como sensibilidad, especificidad, valor predictivo positivo y negativo y precisión (accuracy). Para el análisis se utilizó el paquete estadístico R versión 3.3.1 y la interfaz R Studio versión 0.99.903.

\section{RESULTADOS}

En la base de datos del RT de la Fundación Trauma, con datos de más de 5 años de 10 hospitales de la provincia de Buenos Aires, se identificaron sobre 12021 hechos cerrados 470 pacientes (4\%) con 2 o más lesiones con severidad AIS 3 o superior (Flow Chart 1).

259 de estos pacientes cumplieron con los criterios de politrauma de acuerdo a la definición de Berlín. ${ }^{8}$ La mortalidad en este grupo de pacientes fue $46 \%$, significativamente superior a la del grupo con 2 lesiones AIS3+ pero sin ninguno de los otros cinco criterios auxiliares $\left(19 \%, \mathrm{p}<0.001, \mathrm{chi}^{2}\right)$. 


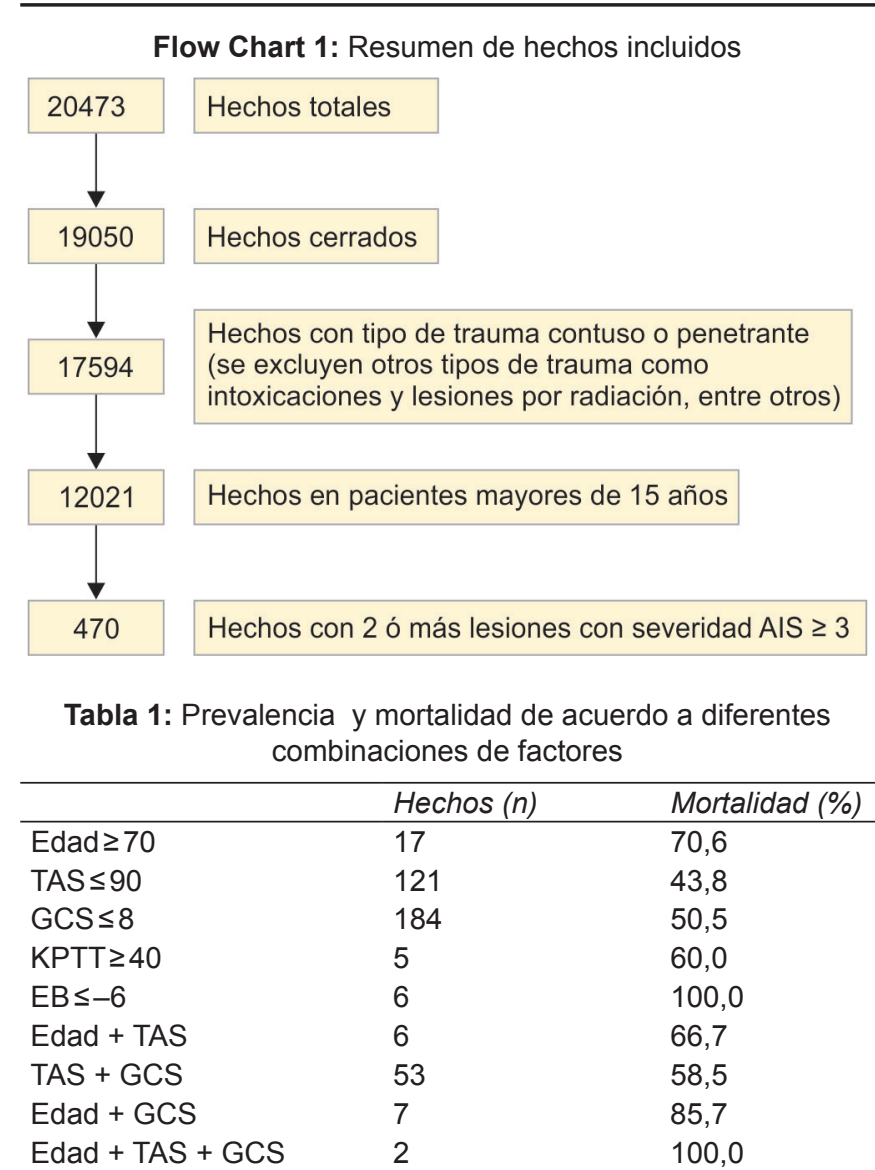

TAS: Tensión arterial sistólica; GCS: Escala de coma de Glasgow; KPTT: Tiempo de tromboplastina parcial; EB: Exceso de base

De todos los componentes complementarios, aislados y combinados los más comunes fueron el GCS y la TAS (Tabla 1). Se aclara que mientras que la TAS, la edad y el score de coma de Glasgow están incluidos desde el inicio de la actividad del registro de trauma, las alteraciones en la coagulación y en el equilibrio ácido base, al momento de realizar este análisis sólo llevaban registrándose 9 meses.*

La mediana de edad fue 29 años (rango 16-91) y el $81 \%$ fueron de sexo masculino. La edad fue diferente en ambos géneros: mujeres, 40 (16-91) vs. varones, 26 (16-88), $\mathrm{p}<0.001$ (Mann-Whitney). En el grupo de menos de 75 la relación varón:mujer fue de 4.4:1, mientras que en el de mayores de 74, fue 0.5:1.

Los mecanismos lesionales ligados al transporte representaron el $75 \%$ de todos los hechos. En los lesionados por transporte, el mecanismo más común fue la motocicleta ( $43 \%$ del total y el $58 \%$ de los mecanismos de transporte). Las agresiones, que en la muestra general representaron una proporción similar a la de motociclistas, en el grupo de politrauma, sólo correspondieron al $8 \%$ del total. La mortalidad fue superior en peatones y caídas (Tabla 2).

El 78\% de los hechos ingresó con algún grado de inestabilidad (según apreciación subjetiva de los médicos tratantes) y esto influyó claramente en la mortalidad: $35.5 \%$ en pacientes estables $(n=31), 39.5 \%$ en inestables $(n=124)$, $60.7 \%$ en críticos ( $n=76)$ y $100 \%$ en PCR (sólo un caso).
Tabla 2: Prevalencia y mortalidad de acuerdo al mecanismo lesional (CIE10, capítulo XX)

\begin{tabular}{llll}
\hline & $\begin{array}{l}\text { Hechos } \\
(n)\end{array}$ & $\begin{array}{l}\text { Hechos (\% } \\
\text { del total) }\end{array}$ & $\begin{array}{l}\text { Mortalidad } \\
(\%)\end{array}$ \\
\hline Motociclista & 111 & 42.9 & 42,3 \\
Peatón & 37 & 14.3 & 70,3 \\
Caídas & 28 & 10.8 & 64,3 \\
Automóvil & 22 & 8.5 & 31,8 \\
Agresión interpersonal & 21 & 8.1 & 33,3 \\
Ciclista & 13 & 5.0 & 38,5 \\
Intención no determinada & 6 & 2.3 & 16,7 \\
Autoagresiones & 5 & 1.9 & 20,0 \\
Camioneta & 3 & 1.2 & 66,7 \\
Otras lesiones por transporte & 3 & 1.2 & 66,7 \\
F. mecánicas inanimadas & 3 & 1.2 & 0,0 \\
Autobús & 2 & 0.8 & 50,0 \\
Otras lesiones por transporte & 2 & 0.8 & 0,0 \\
terrestre & & & \\
F. mecánicas animadas & 1 & 0.4 & 100,0 \\
Fuego, humo y llamas & 1 & 0.4 & 100,0 \\
Fuerzas de la naturaleza & 1 & 0.4 & 0,0 \\
\hline Total & 259 & 100.0 & 45,9 \\
\hline
\end{tabular}

Tabla 3: Scores de trauma de acuerdo a la condición al egreso hospitalario

\begin{tabular}{llll}
\hline $\begin{array}{l}\text { Variable } \\
(n, \text { media, DS) }\end{array}$ & Sobrevivientes & No sobrevivientes & $p$-value \\
\hline RTS & $97,5.796(1.16)$ & $82,4.859(1.48)$ & 0.001 \\
ISS & $140,26.2(8.4)$ & $118,30.6(10.7)$ & 0.001 \\
NISS & $140,30.2(9.5)$ & $118,30.6(10.7)$ & 0.001 \\
Ps por TRISS & $85,0.805(0.17)$ & $79,0.614(0.26)$ & 0.001 \\
\hline
\end{tabular}

RTS: Revised trauma score, ISS: Injury severity score; NISS: New injury severity score; Ps: Probabilidad de sobrevida; TRISS:

Trauma and injury severity score; DS: Desvío standard

El 79\% de los pacientes ingresó al hospital llevado por el sistema prehospitalario de emergencias. El resto fue llevado por policía $(2 \%)$, bomberos $(2 \%)$ o particulares (4\%). Este dato estuvo ausente en $13 \%$ de los ingresos.

El RTS pudo calcularse en el $69 \%$ de los hechos (5.37, 1.39; media y DS) y se relacionó estadísticamente con la supervivencia. Los scores anatómicos (99.6\% de los hechos) y la probabilidad de sobrevida estimada por TRISSS (63\% de los hechos) tuvieron un comportamiento similar (Tabla 3).

El cociente O/E para la estimación de la sobrevida ajustada por riesgo fue 0.727 (85/116.9).

Los pacientes fallecidos tuvieron una estadía significativamente inferior (mediana, RIC): 1 (1-1) vs. 24 (10-40), $\mathrm{p}<0.001$ (test de rangos de Wilcoxon).

El tiempo hasta la muerte (mediana, RIC) fue 4 horas $(1,1-24)$; o sea, el 75\% de los no sobrevivientes falleció en las primeras 24 horas. La principal causa de muerte fueron las lesiones graves del SNC (35\%) con un tiempo hasta la muerte estimado en 10 horas desde el ingreso al hospital.

El 90\% de los pacientes fue atendido en el servicio de Emergencias al ingresar a los hospitales. Una proporción 
Evaluación de la nueva definición de politrauma en una cohorte de pacientes de 10 hospitales argentinos

Tabla 4: Distribución por región corporal y severidad de acuerdo a la codificación por sistema AIS 05

\begin{tabular}{|c|c|c|c|c|c|c|c|c|}
\hline Región corporal & 1 & 2 & 3 & 4 & 5 & 6 & 9 & Total \\
\hline Cabeza & 41 & 123 & 283 & 37 & 37 & 1 & 1 & 523 \\
\hline Cara & 61 & 29 & 11 & 1 & & & & 102 \\
\hline Cuello & 1 & 2 & 2 & & & & & 5 \\
\hline Tórax & 35 & 91 & 204 & 27 & 9 & & 2 & 368 \\
\hline Abdomen & 28 & 61 & 31 & 9 & 13 & & 1 & 143 \\
\hline Columna & & 18 & 3 & 1 & 14 & 1 & 2 & 39 \\
\hline Miembro inferior, pelvis y nalgas & 26 & 94 & 96 & 14 & 2 & & 3 & 235 \\
\hline Miembro superior & 22 & 88 & 4 & 1 & & & 4 & 119 \\
\hline Externo & 4 & & & & 1 & & & 5 \\
\hline Otros tipos de trauma & & & & 1 & & & & 1 \\
\hline Total & 218 & 506 & 634 & 91 & 76 & 2 & 13 & 1540 \\
\hline
\end{tabular}

Tabla 5: Características de los hechos de acuerdo su inclusión en diferentes clasificaciones de trauma grave

\begin{tabular}{lllll}
\hline & $\begin{array}{l}\text { Politrauma } \\
(n=259)\end{array}$ & $\begin{array}{l}\text { Trauma múltiple } \\
(n=802)\end{array}$ & $\begin{array}{l}\text { Trauma mayor (ISS) } \\
(n=1349)\end{array}$ & $\begin{array}{l}\text { Trauma mayor } \\
(\text { NISS })(n=2143)\end{array}$ \\
\hline Género masculino, $\mathrm{n}(\%)$ & $209(81)$ & $656(82)$ & $1141(85)$ & $1848(86)$ \\
Edad, mediana (RIC) & $29(22-43)$ & $29(22-43)$ & $28(21-41)$ & $28(22-41)$ \\
Mortalidad, $\mathrm{n}, \%(I C 95 \%)$ & $119,45.9(40-52)$ & $202,25.2(22-28)$ & $430,31.9(29-34)$ & $525,24.5(23-26)$ \\
ISS, mediana (RIC) & $26(22-34)$ & $18(13-25)$ & $22(17-25)$ & $17(13-25)$ \\
NISS, mediana (RIC) & $27(27-38)$ & $22(17-27)$ & $27(22-34)$ & $22(17-27)$ \\
RTS, media (DS) & $5.37(1.4)$ & $6.67(1.5)$ & $6.45(1.6)$ & $6.76(1.5)$ \\
Estadía hospitalaria en S, mediana (RIC) & $25(10-40)$ & $12(4-29)$ & $13(5-30)$ & $10(4-26)$ \\
Estadía hospitalaria en NS, mediana (RIC) & $1(1-1)$ & $1(1-1)$ & $1(1-1)$ & $1(1-1)$ \\
TEC AIS >=3 & $196(75.7)$ & $387(48.3)$ & $737(54.6)$ & $1180(55.1)$ \\
O/E & 0.727 & 0.833 & 0.807 & 0.844 \\
\hline RIC: Ran
\end{tabular}

RIC: Rango intercuartil; ISS: Injury severity score; NISS: New injury severity score; RTS: Revised trauma score; S: Sobrevivientes; NS: No sobrevivientes; TEC: Traumatismo encéfalocraneano; AIS: Abbreviated injury scale; O/E: Razón de sobrevivientes observados y esperados

baja $(7 \%)$ fue atendida directamente en la Unidad de Cuidados Intensivos como servicio de ingreso al hospital. Un dato significativo fue la baja tasa de ingresos a la UCI luego de la atención inicial. En total sólo se registraron 19 ingresos $(7,5 \%)$.

Se registraron 1540 lesiones en 259 pacientes con politrauma (Tabla 4): 6 (4-7) (mediana y RIC). La región más afectada fue claramente la cabeza (34\% de todas las lesiones). El 76\% de los pacientes con politrauma $(n=196)$ tuvo al menos una lesión en la cabeza con AIS $\geq 3$. La evaluación de la sobrevida ajustada por riesgo (TRISS) en pacientes con y sin lesiones severas en la cabeza fue discretamente más favorable para el primer grupo que para el segundo (O/E): 0.76 vs. 0.63 (con IC95\% superpuestos).

Las lesiones fueron en su gran mayoría serias $(\mathrm{AIS}=3)$. Sólo se registró un $0,8 \%$ de lesiones con severidad indeterminada.

Cuando se compararon en esta muestra los diferentes scores de severidad, los resultados mostraron que el 100\% de los pacientes con politrauma tuvo ISS y NISS mayor o igual a 16 y el $85 \%$ tuvo trauma múltiple; el $28 \%$ de los pacientes con trauma múltiple tuvo politrauma, el $80 \%$ tuvo NISS mayor o igual a 16 y el $68 \%$ tuvo ISS mayor o igual a 16. Entre otras características (Tabla 5), el traumatismo endocraneano tuvo una representación mayor bajo el criterio de politrauma.
Tabla 6: Medidas de performance de las clasificaciones de trauma grave

\begin{tabular}{lllll}
\hline & Politrauma & T. múltiple & ISS $\geq 16$ & NISS $\geq 16$ \\
\hline Sensibilidad & 27.4 & 41.2 & 58.2 & 71.0 \\
Especificidad & 84.8 & 60.3 & 91.0 & 84.2 \\
VPP & 45.7 & 32.7 & 31.9 & 24.5 \\
VPN & 71.4 & 68.6 & 96.8 & 97.6 \\
Precisión & 66.5 & 54.2 & 88.8 & 83.3 \\
\hline
\end{tabular}

VPP: Valor predictivo positivo; VPN: Valor predictivo negativo

La evaluación de performance (Tabla 6) mostró que TM_NISS tuvo la mayor sensibilidad y el mayor VPN, mientras que PT tuvo el mayor VPP. Como medida global, TM_ISS tuvo una precisión superior al resto (89\%) y una especificidad de $91 \%$.

La comparación con las otras definiciones mostró diferencias estadísticamente significativas en mortalidad pero no en ISS, NISS ni RTS. La evaluación de performance mostró que TM_NISS tuvo la mayor sensibilidad y el mayor VPN, mientras que PT tuvo el mayor VPP. Como medida global, TM_ISS tuvo una precisión superior al resto $(89 \%)$ y una especificidad de $97 \%$.

\section{DISCUSIÓN}

En este análisis de la aplicación de la definición de PT a una cohorte de pacientes traumatizados atendidos en 10 hospitales de la Argentina se observó que los 
componentes accesorios a las dos lesiones anatómicas severas tuvieron un desempeño muy diferente para la predicción de mortalidad. La edad avanzada, coagulopatía y acidosis fueron los predictores que se asociaron a una mortalidad superior al 50\%, mientras que la hipotensión y la alteración del nivel de conciencia estuvieron asociados a una mortalidad $\leq 50 \%$.

A diferencia de otras series ${ }^{13,14}$ la proporción de caídas como mecanismo lesional fue baja, predominando claramente los mecanismos ligados al tránsito $(74.5 \%$ de la muestra). Este aspecto pone en duda la comparabilidad del desempeño de los scores en virtud de substanciales diferencias en el case mix de pacientes traumatizados atendidos. Un ejemplo publicado recientemente sobre atención del trauma múltiple en la región de Navarra pone de manifiesto la transición entre dos períodos históricos ligados a un mejor control de las lesiones motovehiculares, lo que modificó los mecanismos lesionales prevalentes de manera substancial. ${ }^{15}$ La escasez de información de países de América Latina sobre estos factores impide poner en contexto lo observado en esta muestra.

Con respecto al análisis de definiciones de trauma severo, el uso de PT no mostró mayor precisión con respecto a otras definiciones usadas previamente (trauma múltiple, trauma mayor). Algunas razones para esto pueden ser que el case mix de pacientes de paísses de medianos y bajos ingresos es claramente diferente del de países desarrollados (en la muestra usada para la definición de $\mathrm{PT}$, la proporción de pacientes con 70 o más años fue de 13\%, mientras que en la aquí reportada fue $6.5 \%$ y la proporción de pacientes con hipotensión, $29 \%$ y $53 \%$, respectivamente), al igual que los resultados esperables de la asistencia (mortalidad en pacientes con ISS $\geq 16$ entre 9 y 15\%, ${ }^{16}$ y en esta muestra, de 32\%). De acuerdo a criterios preestablecidos ${ }^{7}$ la mejor definición de esta entidad debería ser: (1) reproducible, sensible y específica, (2) disponible desde etapas tempranas de la asistencia, y (3) capturar los aspectos fisiológicos y anatómicos del impacto del trauma. En relación a los mismos, el criterio de tener un ISS $\geq 16$ fue el que mejor desempeño tuvo.

Las limitaciones de este estudio fueron: contar con un case mix claramente diferente de la población de referencia para la evaluación de estos scores, no contar con los datos fisiológicos del 31\% de los hechos, no contar con datos para el cálculo de la probabilidad de sobrevida por TRISS en el 37\% de los pacientes, ni con los datos de coagulopatía ni de acidosis desde el inicio del registro, dado que no formaban parte del RT. Estos factores pueden haber llevado a que la muestra sobre la cual se trabajó esté subestimada, y a que el ajuste de los diferentes índices tuviera variaciones con respecto a lo reportado, de contar con la muestra completa.
Como se ha mencionado previamente, ${ }^{17}$ el término politrauma puede encontrarse en una variedad de publicaciones europeas en referencia a lesiones simultáneas que ponen en riesgo la vida, pero muy poco se ve en publicaciones norteamericanas, donde se prefieren los términos "trauma mayor" o "trauma múltiple," para los cuales "politrauma" en ocasiones se usa como sinónimo. ${ }^{18}$ Poco es lo que ha cambiado desde la publicación de la definición revisada de politrauma. Si bien desde el momento de su publicación, el término "polytrauma" fue incluido en el título de 136 publicaciones, ${ }^{19}$ sólo 32 incluyeron la cita de la definición revisada en sus referencias. ${ }^{20}$ Una razón para esto puede tener que ver con que esta definición no ha sido aún incluida dentro del buscador de términos médicos más utilizado (MeSH, PubMed) donde "Polytrauma" no ha sido reconocido aún como término $\mathrm{MeSH}$, estando incluido todavía bajo el término MeSH “Multiple Trauma."21

\section{CONCLUSIONES}

La definición de PT para identificación de pacientes con mayor riesgo de muerte no mostró en esta cohorte ser superior a otras definiciones de uso corriente. Consideramos que la definición de PT podría no ser la misma en el contexto de países desarrollados que en países de medianos y bajos recursos, por lo que debería ser validada en estudios multicéntricos que tengan en cuenta el desarrollo socioeconómico de las regiones intervinientes.

\section{REFERENCIAS}

1. Border JR, LaDuca J, Seibel R. Priorities in the management of the patient with polytrauma. Prog Surg 1975;14:84-120.

2. Tscherne H, Oestern HJ, Sturm JA. Die Belastbarkeit Mehrfachverletzter und ihre Bedeutung für die operative Versorgung. Langenbecks. Arch Chir 1984;364:71-77.

3. Oestern HJ.; Regel G. Clinical care of the polytrauma patient. In: Tscherene H, Regel G, editors. Polytrauma Management. New York: Springer; 1997. p. 225-238.

4. Tuttle MS, Smith WR, Williams AE, Agudelo JF, Hartshorn CJ, Moore EE, Morgan SJ. Safety and efficacy of damage control external fixation versus early definitive stabilization for femoral shaft fractures in the multiple-injured patient. J Trauma 2009 Sep;67(3):602-605.

5. Stahel PF, Smith WR, Moore EE. Current trends in resuscitation strategy for the multiply injured patient. Injury 2009 Nov;40 (Suppl 4):S27-S35.

6. Maier M, Wutzler S, Lehnert M, Szermutzky M, Wyen H, Bingold T, Henrich D, Walcher F, Marzi I. Serum procalcitonin levels in patients with multiple injuries including visceral trauma. J Trauma 2009 Jan;66(1):243-249.

7. Butcher N, Balogh ZJ. The definition of polytrauma: the need for international consensus. Injury 2009 Nov; 40 (Suppl 4):S12-S22.

8. Pape HC, Lefering R, Butcher N, Peitzman A, Leenen L, Marzi I, Lichte P, Josten C, Bouillon B, Schmucker U, et al. The definition of polytrauma revisited: an international consensus process and proposal of the new 'Berlin definition'. J Trauma Acute Care Surg 2014 Nov;77(5):780-786.

9. Butcher NE, Balogh ZJ. The practicality of including the systemic inflammatory response syndrome in the definition of 
polytrauma: experience of a level one trauma centre. Injury 2013 Jan;44(1):12-17.

10. MacKenzie EJ, Rivara FP, Jurkovich GJ, Nathens AB, Frey KP Egleston BL, Salkever DS, Scharfstein DO. A national evaluation of the effect of trauma-center care on mortality. N Engl J Med 2006 Jan;354(4):366-378

11. Groven S, Eken T, Skaga NO, Roise O, Naess PA, Gaarder C. Long-lasting performance improvement after formalization of a dedicated trauma service. J Trauma 2011 Mar;70(3):569-574.

12. World Health Organization. The Injury Chartbook. A graphical overview of the global burden of injuries. Geneva: WHO; 2002.

13. Wong TH, Krishnaswamy G, Nadkarni NV, Nguyen HV, Lim GH, Bautista DC, Chiu MT, Chow KY, Ong ME. Combining the new injury severity score with an anatomical polytrauma injury variable predicts mortality better than the new injury severity score and the injury severity score: a retrospective cohort study. Scand J Trauma Resusc Emerg Med. 2016 Mar;24:25-35.

14. Konjo H, Švraka E, Omerović D, Avdić D. Incidence of hospital mortality in polytrauma patients in a tertiary center in Bosnia and Herzegovina. Journal of Health Sciences 2016;6(1):67-71.
15. Gradin Purroy C, Belzunegui Otano T, Bermejo Fraile B, Teijeira R, Fortún Moral M, Reyero Díez D. Changes in the characteristics and incidence of multiple-injury accidents in the Navarre community over a 10 -year period. Emergencias 2015;27:174-180.

16. Wong TH, Lumsdaine W, Hardy BM, Lee K, Balogh ZJ. The impact of specialist trauma service on major trauma mortality. J Trauma Acute Care Surg 2013 Mar;74(3):780-784.

17. Butcher N, Balogh ZJ. The definition of polytrauma: the need for international consensus. Injury 2009 Nov;40 (Suppl 4): S12-S22.

18. Sikand M, Williams K, White C, Moran CG. The financial cost of treating polytrauma: implications for tertiary referral centres in the United Kingdom. Injury 2005 Jun 36(6): 733-737.

19. Búsqueda en PubMed con este criterio: polytrauma [Title] AND (“2014/11/01"[PDAT]: “2016/10/01”[PDAT]).

20. Datos de búsqueda en Google Scholar, actualizada el 01/10/2016.

21. Búsqueda en PubMed (https://www.ncbi.nlm.nih.gov/ mesh/?term=polytrauma) actualizada el 01/10/2016. 


\section{INVITED COMMENTARY}

Trauma continues to have a high socioeconomic impact on society worldwide since it is the leading cause of death and disability in young patients. ${ }^{1-3}$

In order to guide funds and human resources allocation to tackle public health problems it is of importance to understand what the local issues are. Many underserved places in the world lack registries to help understand the indigenous needs and the impact of trauma in their population.

This article is a great show of Argentinian efforts in defining the highest risk of death for trauma patients.

Although this is a retrospective review and as such has the limitations of that nature, it illustrates important information, as well as the lack of applicability of some concepts accepted worldwide to the South American publication. ${ }^{2}$

I couldn't agree more with the conclusion of the article: "we need to validate definitions in multicenter trials before providing with recommendations." Latin America has an immense population of injured patients with a variety of mechanisms and diversity of local resources. It is the responsibility of all surgeons and scientists to help this population with the development of prospective, international, multicenter studies to better define the issues so we can allocate the necessary resources to the areas in need.

\section{REFERENCES}

1. Lefering R. Development and validation of the revised injury severity classification score for severely injured patients. Eur J Trauma Emerg Surg 2009 Oct;35(5):437-447.

2. Pape HC, Lefering R, Butcher N, Peitzman A, Leenen L, Marzi I, Lichte P, Josten C, Bouillon B, Schmucker U, et al. The definition of polytrauma revisited: an international consensus process and proposal of the new 'Berlin definition'. J Trauma Acute Care Surg 2014 Nov;77(5):780-786.

3. Pape HC, Probst C, Lohse R, Zelle BA, Panzica M, Stalp M, Steel JL, Duhme HM, Pfeifer R, Krettek C, et al. Predictors of late clinical outcome following orthopedic injuries after multiple trauma. J Trauma 2010 Nov;69(5):1243-1251.

Paula Ferrada

Associate Professor of Surgery

Medical Director, Surgical and Trauma ICU

Director, Surgical Critical Care Fellowship Virginia Commonwealth University, Richmond, Virginia 


\section{INVITADO COMENTARIO}

\section{Evaluación de la Nueva Definición de Politrauma en una Cohorte de Pacientes de 10 Hospitales Argentinos}

El trauma continúa teniendo un alto impacto socioeconómico en la sociedad en todo el mundo ya que es la causa principal de muerte y discapacidad en pacientes jóvenes. ${ }^{1-3}$

A fin de orientar la asignación de fondos y recursos humanos para abordar los problemas de salud pública, es importante comprender cuáles son los problemas locales. Muchos lugares desatendidos en el mundo carecen de registros para ayudar a comprender las necesidades indígenas y el impacto del trauma en su población.

El manuscrito actual es una gran muestra de los esfuerzos de Argentina para definir el mayor riesgo de muerte para los pacientes con traumatismos.

Aunque se trata de una revisión retrospectiva y, como tal tiene las limitaciones de esa naturaleza, ilustra información importante, así como la falta de aplicabilidad de algunos conceptos aceptados en todo el mundo [de] publicacion[es] sudamericana[s]. ${ }^{2}$

No podría estar más de acuerdo con la conclusión del artículo: We need to validate definitions in multicenter trials before providing with recommendations (Tenemos Que Validar las Definiciones en Ensayos Metacéntricos Antes de Proporcionar Recomendaciones). América Latina tiene una inmensa población de pacientes lesionados con una variedad de mecanismos y diversidad de recursos locales. Es responsabilidad de todos los cirujanos científicos ayudar a esta población con el desarrollo de estudios prospectivos, internacionales y metacéntricos para definir mejor los problemas a fin de poder asignar los recursos necesarios a las áreas que lo necesitan.

\section{REFERENCIAS}

1. Lefering R. Development and validation of the revised injury severity classification score for severely injured patients. European journal of trauma and emergency surgery: official publication of the European Trauma Society. 2009;35(5):437-447.

2. Pape HC, Lefering R, Butcher N, Peitzman A, Leenen L, Marzi I, et al. The definition of polytrauma revisited: An international consensus process and proposal of the new 'Berlin definition'. The journal of trauma and acute care surgery. 2014;77(5):780-786.

3. Pape HC, Probst C, Lohse R, Zelle BA, Panzica M, Stalp M, et al. Predictors of late clinical outcome following orthopedic injuries after multiple trauma. The Journal of trauma. 2010;69(5):1243-1251.

Paula Ferrada MD FACS

Associate Professor of Surgery Medical Director Surgical and Trauma ICU Director Surgical Critical Care Fellowship Virginia Commonwealth University 